\title{
Study on Interaction of 2,3-Butadione Monoxime with some Phenols
}

\author{
A. S. P. Azzouz \\ Chemistry Dept. \\ College of Education \\ Mosul University
}

\author{
A. A. S. Siddieq \\ Chemistry Dept. \\ College of Science \\ Mosul University
}

\author{
N. B. Sharif \\ Chemistry Dept. \\ College of Science \\ Mosul University
}

Received
$01 / 04 / 2007$
Accepted

$03 / 10 / 2007$

Keywords: Interaction, oxime, phenols proton transfer, hydrogen bonding, uv spectra and thermodynamics.

\section{خلاصة}

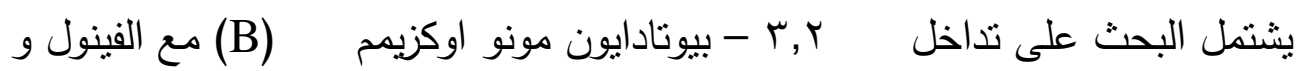

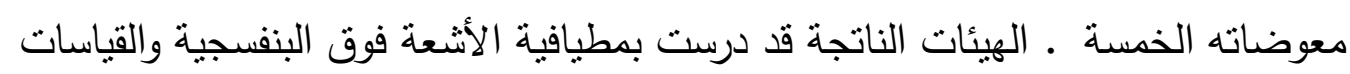

$$
\begin{aligned}
& \text { الثرموديناميكية. } \\
& \text { توصلت الدراسة إلى تكوين أنواع من المبرتتات الأحادية ، المبرتتات الثنائية وكذالك }
\end{aligned}
$$

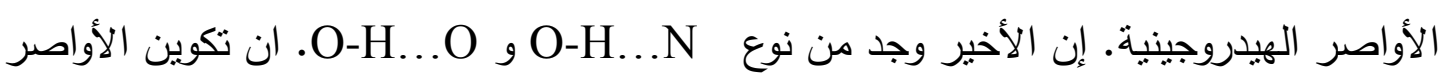

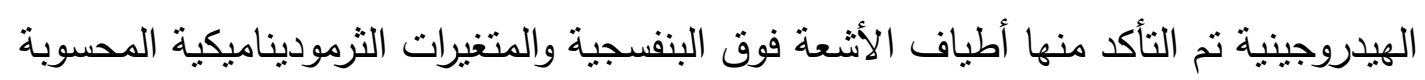

$$
\begin{aligned}
& \text { والتي بينت إن إنشارات هH و ه هي سالبة بالإضلفة إلى قيمهما. }
\end{aligned}
$$

\begin{abstract}
The interaction of 2, 3-butadione monooxime (B) with phenol and its five substituents in n-heptane are studied by using uv spectroscopy. The study lead to the formation of mono-protonated, bi-protonated as well as intramolecular hydrogen bonding species. The later are found to be of the type $\mathrm{O}-\mathrm{H} \cdots \mathrm{N}$ and $\mathrm{O}-\mathrm{H} \cdots \mathrm{O}$. The process of hydrogen bond formation is confirmed here from uv spectra and the calculated thermodynamic parameters. This shows that $\Delta \mathrm{H}$ and $\Delta \mathrm{S}$ have a negative signs, in addition to their values which support hydrogen bond formation.
\end{abstract}




\section{Introduction}

In visual pigment the aldehyde is condensed with an amino group of specific lysine residue forming Schiff base as a best evidence indicates the Schiff base is acidified ${ }^{(1)}$. Several studies were reported ${ }^{(2-5)}$ regarding hydrogen bond formation and proton transfer in a certain Schiff bases.

Takemura et $\mathrm{al}^{(6)}$ found that addition of phenol to 3-methylpentane solution of all trans retinal at $77 \mathrm{~K}$ causes a shift of wave length from 383 to $424 \mathrm{~nm}$ and also causes changes in excitation and fluorescence spectra.

In this laboratory, the effect of hydrogen bonding on the stability constant values ${ }^{(7-8)}$, kinetics of the decomposition of Schiff base $-\mathrm{AlCl}_{3}$ complex ${ }^{(9)}$, the uv of absorption spectra of cinnamylidene n-butylamine with 4-substituents phenols ${ }^{(10)}$, thiophenol and p-thiocresol ${ }^{(11)}$ are examined.

The present investigation is an extension of the last two studies. It deals with the effect of phenol and its substiuents such as o-chloro, mchloro, p-chloro, p-methoxy and 2, 4-dimethylphenol, on the uv absorption spectrum of $\mathrm{B}$ dissolved in $\mathrm{n}$-heptane.

\section{Experimental}

All chemical used throughout this work are supplied from Fluka origin. They are used as supplied without any further purification with exception of phenol and 2, 4-dimethylphenol. The last two were purified by fractional distillation. Pure phenol and 2, 4-dimethylphenol have b.p's of $70^{\circ} \mathrm{C}$ (12 torr) and $90^{\circ} \mathrm{C}$ (10 torr) respectively. Crude n-heptane was purified by standard method ${ }^{(12)}$. 2, 3-Butadioneoxime (B) was synthesized using the standard method ${ }^{(5,12-13)}$.

The uv spectra are measured using Shimdzu computerized double beam model UV-1601 by using matched $1 \times 1 \times 3 \mathrm{~cm}^{3}$. All uv spectra were measured against phenolic blank solutions. Also IR spectra were measured for solid samples using computerized Tensor -27 and by $\mathrm{KBr}$ disk method. The linear plots were performed by using Excel computer program.

\section{Results and Discussion}

The solid IR spectra of oxime B shows a strong doublet bands at wave numbers $1659.5(\mathrm{~s}) \mathrm{cm}^{-1}$ and $1664.0(\mathrm{~s}) \mathrm{cm}^{-1}$. Other bands are also observed at wave numbers $3063(\mathrm{~m}) \mathrm{cm}^{-1}$ and $3316(\mathrm{~b}) \mathrm{cm}^{-1}$. They are assigned $^{(14)}$ to azomethine linkage of oxime, the conjugated carbonyl group, the $\mathrm{C}-\mathrm{H}$ stretching vibration and hydrogen bonding respectively. To confirm the type of hydrogen bonding in B and in last band, the 
dilution method ${ }^{(15,16)}$ is followed and after measuring IR spectrum of $\mathrm{B}$ solution of concentrations $1 \times 10^{-3} \mathrm{M}$ and $0.5 \times 10^{-4} \mathrm{M}$ in $\mathrm{CCl}_{4}$ solvent. These measurements clearly show the existence of stable six membered intramolecular hydrogen bonding.

Association reactions are responsible on many phsio-chemical properties such as stability ${ }^{(17)}$ of system and change in absorption ${ }^{(1,5)}$ spectra. This encourage the workers to deal with the interactions of compound B with phenol and its substituents.

The preliminary uv spectrum of compound $B$ in a purified $n-$ heptane shows a main absorption band at wavelength of $218.5 \mathrm{~nm}$ with molar extinction coefficient value of 8470 Liter. mole . $\mathrm{cm}^{-1}$. This was followed by recording a set of uv spectra of fixed $10^{-4} \mathrm{M}$ of concentration of $\mathrm{B}$ with a variable concentrations of soluble phenolic compounds as a phenol, o, m, p-chlorophenols, p-methoxyphenol and 2,4-dimethylphenol. The concentrations of the latter are in the range between $(1-9) \times 10^{-4} \mathrm{M}$. This gives a relative concentration between different phenols $/ B$ in a range of ratio values $1: 1-9: 1$, or phenols are mostly in excess concentrations as compared to the concentration of $\mathrm{B}$, during uv measurements. This gives the following results.

i- The normal spectrum of $\mathrm{B}$ shows a single absorption band as in Fig.1a. Addition of phenol, o-chlorophenol and m-chlorophenol resulted to the appearance of two new absorptions located at higher wavelengths when compared to the uv spectrum of $\mathrm{B}$. The last is started when the concentrations of phenol, o-chlorophenol and $\mathrm{m}$ chlorophenol over concentration of $\mathrm{B}$ of a relative values of 3:1, 5:1 and 2:1 respectively as in Fig.1b after addition of m-chlorophenol as a typical example. Below these values again the normal spectra of $\mathrm{B}$ is only recorded.

ii- On using the para substituted phenols as p-chlorophenol, pmethoxyphenol and p-bromophenol similar results as in (i) is also started to be observed at relative concentration ratios of these phenols of a values of 3:1, 5:1 and 2:1 respectively. Increasing the relative concentration of these phenols above the last stated values, resulted in appearance of a fourth band in each case located at shorter wavelength when compared to the weak intensities absorptions mentioned in (i).In other words, the uv spectrum of B is now composed from four uv absorptions. Two of these are bands of reasonable and variable intensities and two of weaker absorption intensities. Fig.1C shows the uv absorption spectrum for a mixture of $10^{-4} \mathrm{M} \mathrm{B}$ with $4 \times 10^{-4} \mathrm{M}$ of $\mathrm{p}$-chlorophenol in heptane as typical example. 
These results can be interpreted by the following facts:

1) In case of (i) a protonated ${ }^{(2-5)}$ oxime is formed by proton transfer reaction from donor phenol molecules to an acceptor oxime molecule which contains a hetero oxygen and nitrogen atoms. These can form either oxonium or nitrilium ions ${ }^{(18)}$ respectively as a monoprotonated oxime forms. These ions are in agreement ${ }^{(18)}$ with monoprotonated oxime formed by the reaction of B molecule with $\mathrm{p}$ chlorophenol and p-methoxyphenol resulting to the appearance of double uv absorptions of weak intensities as in Fig.1b.

To support the idea of protonated oxime form, this the uv spectrum of B after addition of two drops of trifluro acetic acid or trichloro acetic acid in heptane is measured. This gives again a weak intensity absorptions of protonated oxime form of wavelength $294 \mathrm{~nm}$ or $283 \mathrm{~nm}$ respectively. The reason for the last weak absorptions is due to the very weak solubility of either the mono protonted oxime or the di protonted oxime form (highly polar) in a non polar heptane solvent.

Now when p-bromphenol is added to the same solution of $\mathrm{B}$, this result to produce the triple new uv absorptions of weak intensities as assigned to the two mono-protonated oxime of type oxonium and nitrilium ions and to the third diprotonated oxime form which contains both oxonium and nitrilium ions. The last double positive charges are in agreement with the kinetic ${ }^{(19)}$ study on reaction of 2-pyridine aldoxime with percholric acid and the hydrolysis ${ }^{(20)}$ reaction of $\beta$-diketone Schiff base.

2) In case of (ii) the addition of phenols in a different successive concentrations to a fixed $10^{-4} \mathrm{M}$ of $\mathrm{B}$ is resulted to the formation of protonated weak absorptions as noticed when concentration of $\mathrm{p}$ chlorohenol, p-methoxyphenol and p-bromophenol over B concentration to have relative values of $3: 1,5: 1$ and $2: 1$ respectively. This is a similar result as observed in previous paragraph. It is in agreement with earlier works ${ }^{(5)}$ performed in this laboratory using phenol and 2,3-dimethylphenol with benzylidene aniline.

3) Themodynamic study:

Increasing the concentration of the same phenolic compounds to a greater ratios of same order of values 4:1, 6:1 and 3:1 are accompanied with the generation of a new additional uv band at the expense of original oxime band. The last band with original band of $\mathrm{B}$ are observed to be in equilibrium to each other and at temperature ranges between $(293-333) \mathrm{K}$ and attributed to the following equilibrium reaction:

Oxime $\mathrm{B}+$ phenols $\rightleftharpoons$ complex molecule or ion 
Now it is important to prove the type of interaction between B and phenols by thermodynamic calculations. These results are collected in Table (1) which shows the following results:-

A) The Gibbs free energy $\Delta \mathrm{G}$ is calculated from equation (1) of the form:-

$$
\Delta \mathrm{G}=-\mathrm{RTLnK}
$$

All $\Delta \mathrm{G}$ thermodynamic parameters of interaction reaction stated above have a positive signs with the exception of interaction of $\mathrm{p}$-chlorophenol at $293 \mathrm{~K}$. The last case is in accordance with our exception of the interaction of $\mathrm{B}$ with p-chlorophenol at temperature mentioned and is spontaneous process. The main other $\Delta \mathrm{G}$ calculated have positive signs or the interaction of phenols with $\mathrm{B}$ are non spontaneous. The last can be interpreted by the following suggestions:-

The interaction of B with p-bromophenol, p-methoxyphenol and p-chloro phenol may occur by two consecutive processes:-

1) The first is the irreversible proton transfer reaction from donor phenols to an accepter B molecule at low relative concentrations ratios of phenols/B as a main step.

2) The second is the reversible interaction between $B$ molecule and the last three phenols, possibly by hydrogen bond formation, which occur at high relative concentrations of phenol as a minor step. Hence these two irreversible and reversible processes may give a positive average $\Delta \mathrm{G}$ values with phenols as in Table (1). At any how, the negative or positive signs of $\Delta \mathrm{G}$ are in full accordance with a similar interaction study ${ }^{(5)}$ of benzil monoxime with phenol.

B) The equilibrium constant $K_{\text {eq }}$ of the interaction reaction stated above is evaluated from uv spectrum and has an inverse relationship with absolute temperature. This facilitates the evaluation of heat of reaction of $\Delta \mathrm{H}$ from Vant Hoff equation of the form as in the equation (2):-

$$
\mathrm{LnK}=\text { constant }-\Delta \mathrm{H} / \mathrm{RT}
$$

By using excel computer program the plots of $\operatorname{lnK}$ versus $\mathrm{T}^{-1}$ for $\mathrm{p}$ chlorophenol, p-methoxyphenol and p-bromophenol, show a good straight lines of $\mathrm{R}^{2}$ values of about 0.95 as in Fig. (2). This allows the acceptable estimation of $\Delta \mathrm{H}$ values.

The calculated heat of reaction $\Delta \mathrm{H}$ for interaction of $\mathrm{B}$ with the last phenols stated later have a negative signs and their range values as $0.472-9.092 \mathrm{~kJ} . \mathrm{mole}^{-1}$ as in Table (1) depending on phenol used or its pKa.

These $\Delta \mathrm{H}$ values just mentioned support ${ }^{(5,15)}$ the interactions processes by hydrogen bonding in the systems under study.

C) The entropy changes $\Delta \mathrm{S}$ of hydrogen bond formation is estimated from the standard equation (3) of a form:- 


$$
\Delta \mathrm{G}=\Delta \mathrm{H}-\mathrm{T} \Delta \mathrm{S}
$$

All $\Delta \mathrm{S}$ values estimated for hydrogen bond formation have a negative signs which agrees with the idea of interactions by hydrogen bonding ${ }^{(15}$.

Finally, both negative signs and values of $\Delta \mathrm{H}$ and $\Delta \mathrm{S}$ thermodynamic parameters are agreed with the several hydrogen bonding studies $^{(5,15,21,22)}$ performed in our laboratory.

\section{Conclusions}

1) The interactions of 2,3-butadione monoxime with phenols are studied in $\mathrm{n}$-heptane by uv spectroscopy and thermodynamic calculations were performed.

2) The stated interactions occurr by two consecutive processes. The first one is the proton transfer reaction from donor phenols to an acceptor oxime as a main irreversible step; the second one is the reverse hydrogen bonding process.

3) The hydrogen bonding process facilitates the estimation of thermodynamic parameters. The negative signs as well as the values of $\Delta \mathrm{H}$ and $\Delta \mathrm{S}$ parameters support the interaction by hydrogen bonding process ${ }^{(5,15)}$.

4) The numbers of protonated oxime is related to the number of weak uv absorptions observed in the spectrum.

5) Mono-protonated ${ }^{(2,5)}$ oxime can be either of the type oxonium or nitrilium ion. Their combinations produce a diprotonated oxime form. This finding is in agreement with previous study ${ }^{(19-20)}$.

6) Protonated oxime is formed at the excess concentrations of all phenols with a relative concentration ratios of ranging values between $2 / 1$ to $5 / 1$, depending on pKa value of phenols.

7) By increasing the relative concentration ratios of p-chlorophenol, p-bromophenol and p-methoxyphenol to a values of 4/1, 3/1 and $6 / 1$ respectively, the formation of intermolecular hydrogen bonding is observed in the interacting system. 

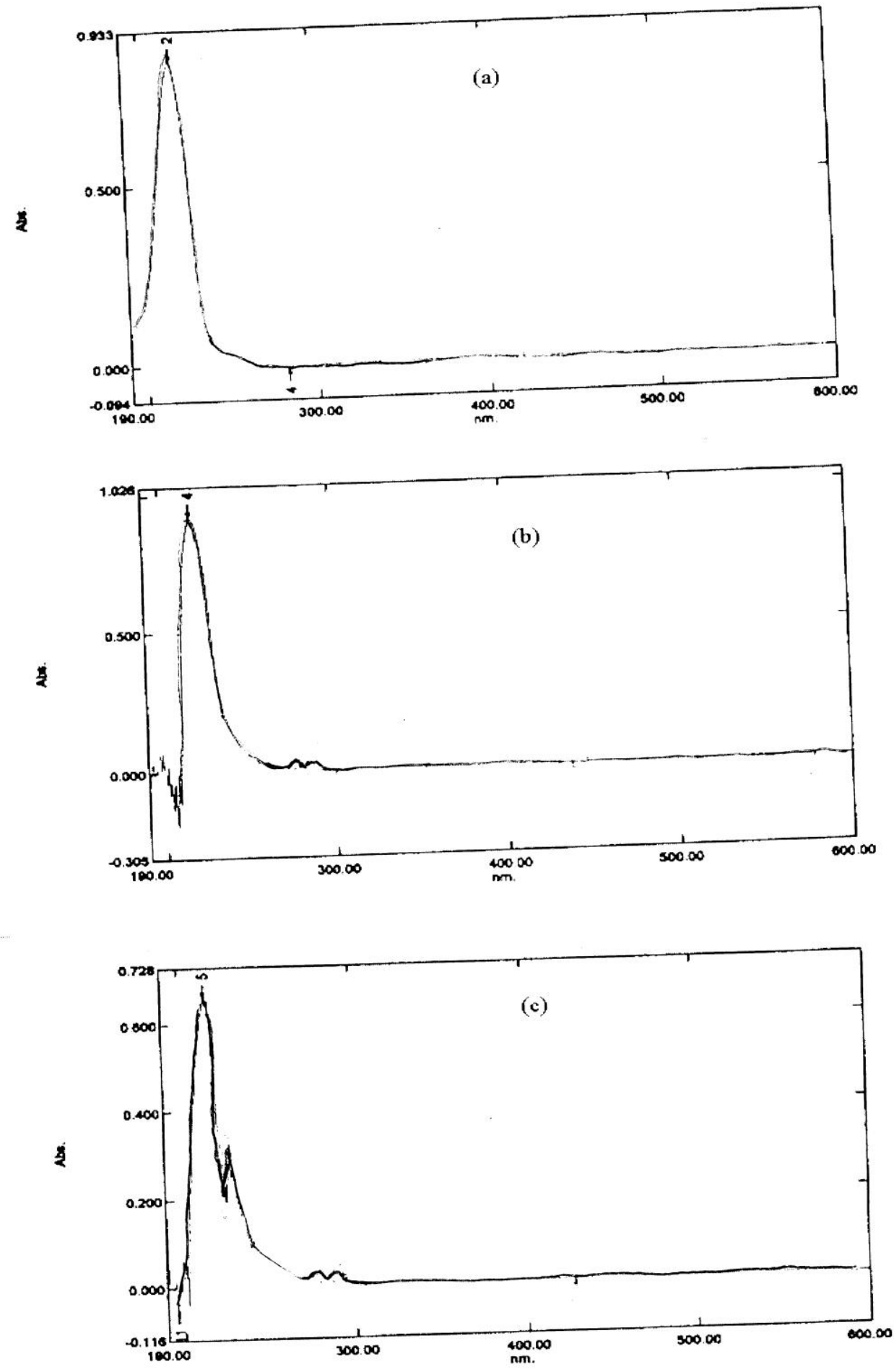

Fig. 1 The UV absorption spectra in n-heptanie for a. 10-4 M 2,3-butadione monoxime (B)

b. Mixture of $10^{-4} \mathrm{M}$ of (B) with $10^{-4} \mathrm{M}$ m-chlorophenol

c. Mixture of $10^{-4} \mathrm{M}$ of (B) with $4 \times 10^{-4} \mathrm{M}$ p-chlorophenol 
Study on Interaction of 2,3-Butadione Monoxime with some Phenols.
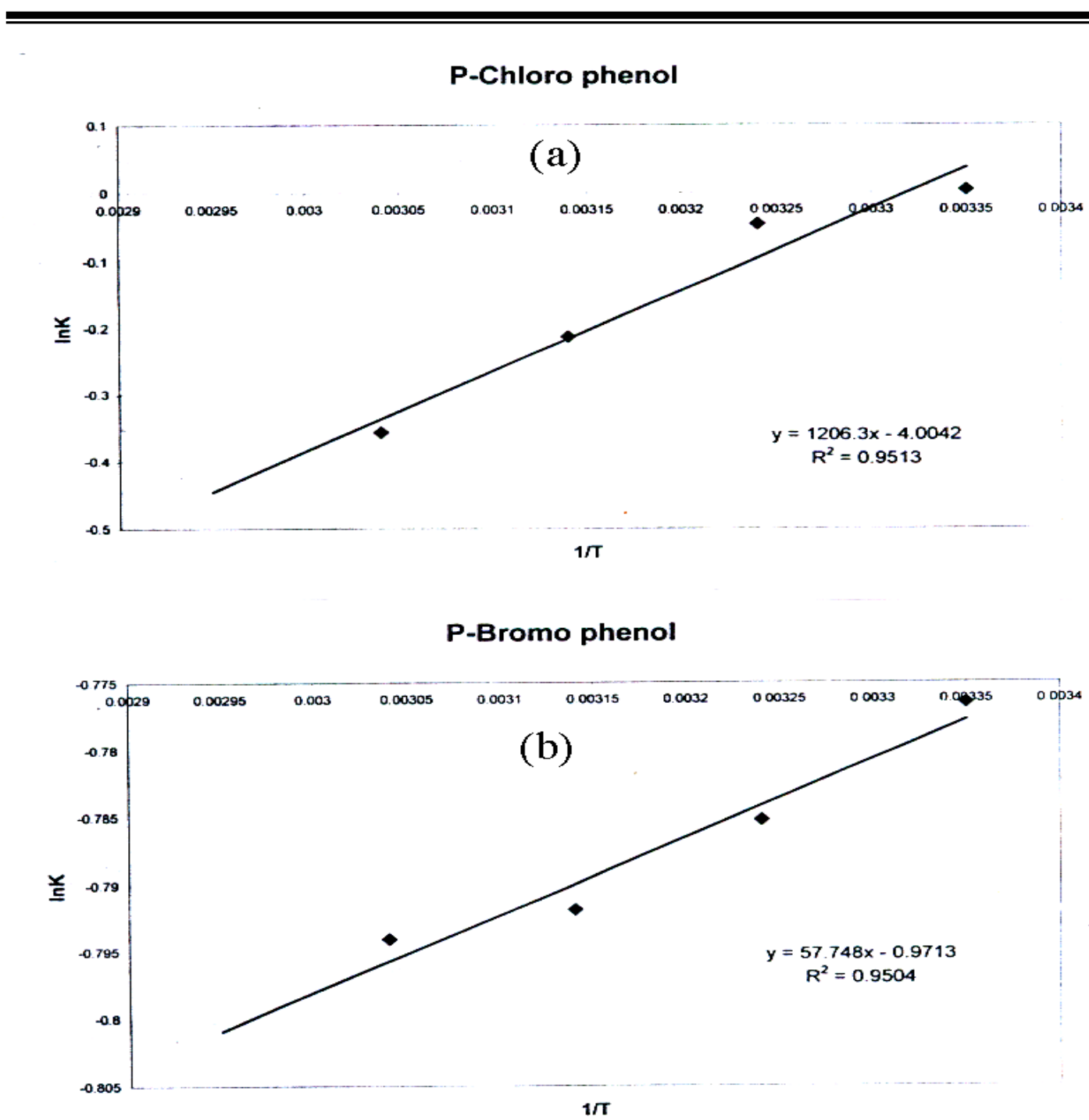

P-Methoxy phenol

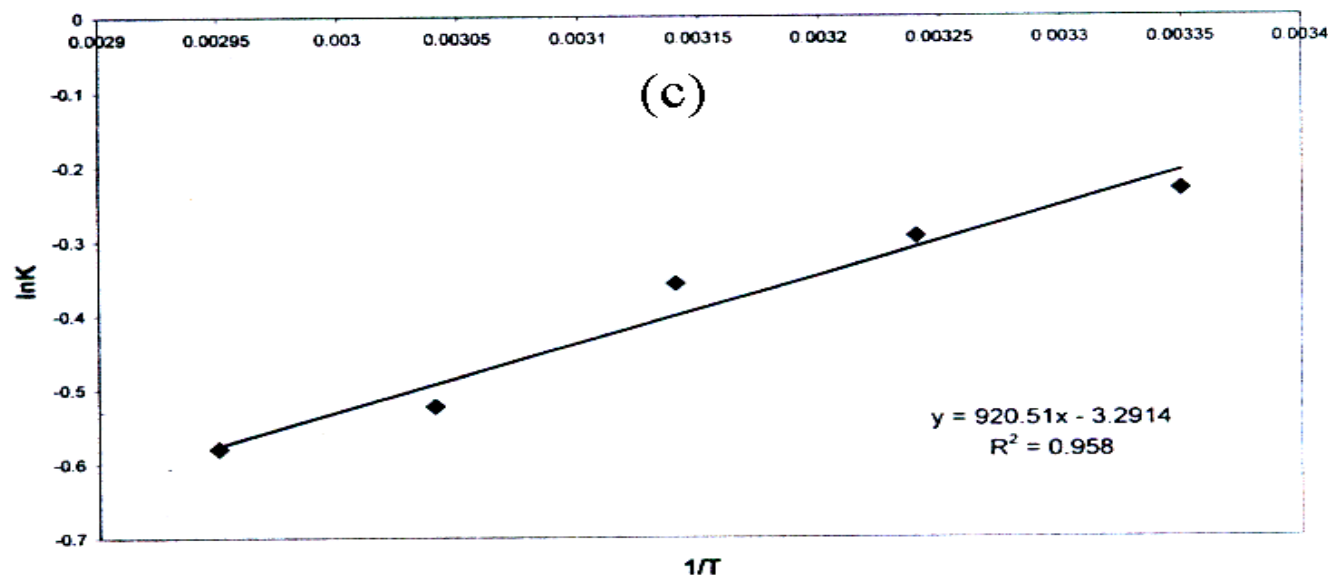

Fig .2 The relationship between InK versus $\mathrm{T}^{-1}$ for the interaction of $\mathrm{B}$ with

a. p-chlorophenol

b. p-bromophenol

c. p-methoxyphenol 
Table (1): Thermodynamic parameters of hydrogen bonding between phenols with 2,3-butadione monoxime.

\begin{tabular}{|c|c|c|c|c|c|}
\hline Phenol & $\begin{array}{c}\text { Temp./ } \\
\mathrm{K}\end{array}$ & $\mathrm{LnK}_{\mathrm{eq}}$ & $\begin{array}{c}\Delta \mathrm{G} \\
\mathrm{kJ} \cdot \mathrm{mole}^{-1}\end{array}$ & $\begin{array}{c}\Delta \mathrm{H} \\
\mathrm{kJ} \cdot \mathrm{mole}^{-1}\end{array}$ & $\begin{array}{c}\Delta \mathrm{S} \\
\mathrm{kJ} \cdot \mathrm{mole}^{-1} \mathrm{~K}^{-1}\end{array}$ \\
\hline \multirow[t]{3}{*}{ 1) p-Bromophenol } & 293 & -0.7765 & +1.89 & -0.475 & -8.071 \\
\hline & 303 & -0.7853 & +1.98 & -0.469 & -8.082 \\
\hline & 313 & -0.7919 & +2.06 & -0.467 & -8.073 \\
\hline & 323 & -0.7941 & +2.13 & -0.476 & -8.063 \\
\hline \multicolumn{6}{|c|}{$\bar{\Delta} \overline{\mathrm{G}}=2.015 \mathrm{~kJ} \cdot \mathrm{mole}^{-1}, \bar{\Delta} \overline{\mathrm{H}}=-0.472 \mathrm{~kJ} . \mathrm{mole}^{-1}, \bar{\Delta} \overline{\mathrm{S}}=-8.074 \mathrm{~kJ} \cdot \mathrm{mole}^{-1} \mathrm{~K}^{-1}$} \\
\hline \multirow[t]{5}{*}{ 2) p-methoxyphenol } & 293 & -0.2326 & +0.567 & -7.451 & -27.3 \\
\hline & 303 & -0.2944 & +0.742 & -7.549 & -27.4 \\
\hline & 313 & -0.3566 & +0.928 & -7.637 & -27.4 \\
\hline & 323 & -0.5200 & +1.396 & -7.442 & -27.4 \\
\hline & 333 & -0.5798 & +1.605 & -7.507 & -27.4 \\
\hline \multicolumn{6}{|c|}{$\bar{\Delta} \overline{\mathrm{G}}=+1.048 \mathrm{~kJ} . \mathrm{mole}^{-1}, \bar{\Delta} \overline{\mathrm{H}}=-07.517 \mathrm{~kJ} . \mathrm{mole}^{-1}, \bar{\Delta} \overline{\mathrm{S}}=-27.4 \mathrm{~kJ} . \mathrm{mole}^{-1} \mathrm{~K}^{-1}$} \\
\hline \multirow[t]{5}{*}{ 3) p-Chlorophenol } & 293 & +0.0399 & -0.097 & -9.851 & -33.9 \\
\hline & 303 & -0.0460 & +0.115 & -9.971 & -33.3 \\
\hline & 313 & -0.2132 & +0.554 & -9.865 & -33.3 \\
\hline & 323 & -0.3566 & +0.957 & -9.795 & -33.3 \\
\hline & 333 & -0.3816 & +1.056 & -10.029 & -33.2 \\
\hline \multicolumn{6}{|c|}{ 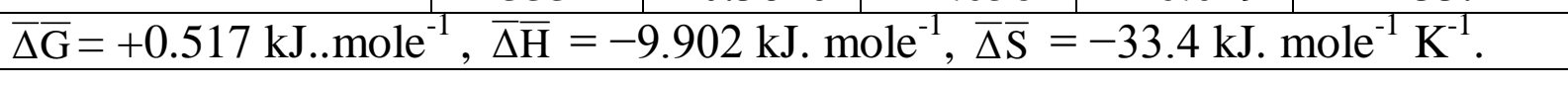 } \\
\hline
\end{tabular}




\section{References}

1) S. K. AL-Dilaimi, J. C. Aumiller, R. H. Johnson and P. E. Baltz, Photochem. Photobiol., 46 (1987) 403.

2) J. Favrot, J. M. Leclerca, R. Roberge, C. Sandrofy and D. Vocelle, Photochem. Photobiol., 29 (1979) 99.

3) D. Vocelle, Rev. Can. Biol., 39 (1980) 247.

4) M. Bissonette, H. Lethanh and D. Vocelle, Can. J. Chem., 62 (1984) 1459.

5) A. S. P. Azzouz, Z. Phys. Chem.., 216 (2002) 1053.

6) T. Takemura, P. K. Das, G. Hug and R. S. Becker, J. Amer. Chem. Soc., 100 (1978) 2626.

7) S. K. AL-Dilaimi and A. A. Mutar, J. Edu. Sci., 2 (1980) 41.

8) S. K. AL-Dilaimi and A. S. P. Azzouz, J. Indian Chem. Soc., LIV, (1977) 678.

9) S. K. AL-Dilaimi and A. T. Hassan, J. Edu. Sci., 6 (1989) 108.

10) S. K. AL-Dilaimi and A. S. P. Azzouz and K. S. Tawfiq, J. Edu. Sci., 10 (1990) 65.

11) S. K. AL-Dilaimi, J. Edu. Sci., 10 (1990) 65.

12) A. I. Vogel, Text Book of Practical Organic Chemistry, $14^{\text {th }}$ Ed., Longman, (1978).

13) A. S. P. Azzouz, Nati. J. Chem., 22 (2006) 214.

14) W. Kemp, Organic Spectroscopy, Macmillan, (1978), pp. 19-52.

15) G. C. Pimental and A. L. Mecellellan, The Hydrogen Bond, Freeman, New York, (1960).

16) A. S. P. Azzouz and S. S. Othman, J. Edu. Sci., 45 (2000) 3.

17) A. Martin, Physical Pharmacy, $4^{\text {th }}$ Ed., Lea and Febiger, (1993), pp. 408-494.

18) M. S. Masoud, G. B. Mohammed, A. M. Hindawey and T. M. Abdel_Fattah, Mutah J. for Res. Stud., 6 (1991) 35.

19) A. S. P. Azzouz and K. A. Abdullah and K. I. Niemi, Mutah J for Res. Stud., 10 (1995) 77.

20) H. Saeed, M. H. Watton and A. W. A. Sultan, Thermochimica Acta, 67 (1983) 17.

21) A. A. S. P. Azzouz, A. A. S. Siddieq and E. Z. Sulyman, J. Edu. Sci., 51 (2001) 83.

22) A. S. P. Azzouz and S. S. Othman, J. Edu. Sci., 48 (2001) 32. 